\title{
Providing quality in anesthesia care in low- and middle-income countries
}

\author{
David J. Wilkinson, MBBS
}

Received: 14 July 2014/ Accepted: 8 August 2014/Published online: 15 August 2014

(C) Canadian Anesthesiologists' Society 2014

At this point in time, there is a major discrepancy in the availability of essential surgery and safe anesthesia between populations in affluent nations and those who live in low- or middle-income countries (LMICs). It has been estimated that only around 3\% of the 234 million surgical operations that take place annually around the globe occur in the poorest one-third of the world's population. Indeed, approximately 2 billion people have no access to any surgical care at all.

Many organizations expend considerable sums of money, time, and personnel involvement in trying to find effective ways to reverse these appalling statistics. Some of these groups - and there are now a myriad of religious, academic, and non-governmental based groups of this nature - provide surgical operations that are sorely needed, often on a cyclical basis. There is almost a sense of "medical tourism" with some of these visits, with no apparent long-term gain for the community apart from the very real benefit to the individuals whose lives have been changed for the better.

Furthermore, solely providing financial incentives to create better care does not always work. A recent editorial

The WFSA is currently involved in the development and financial sponsorship of numerous SAFE courses.

La WFSA est actuellement impliquée dans le développement et le parrainage financier de nombreux cours «SAFE ».

D. J. Wilkinson, MBBS ( $₫)$

World Federation of Societies of Anaesthesiologists, London,

UK

e-mail: lorikeet08@gmail.com

D. J. Wilkinson, MBBS

St Bartholomew's Hospital, Barts Health NHS Trust, London, UK in the New England Journal of Medicine highlighted India's attempt to improve care and decrease maternal mortality by implementing Janani Suraksha Yojana, where mothers were paid money to have their babies delivered in hospitals. The results of this undertaking showed that while there was a spectacular rise in the number of hospital deliveries, maternal mortality remained unchanged. The writers pleaded for quality to be the prime aspect of intervention and ended with the great statement that “....doing more isn't better. Doing better is better". 1

The World Federation of Societies of Anaesthesiologists (WFSA) contends passionately that beyond providing individual successful surgical cases, all teaching and humanitarian aid should have a major component that provides a legacy and sustainability for the resident population. The Canadian Anesthesiologists' Society International Education Foundation (CAS IEF) shares this view and has been very active in Africa and Nepal in recent years. In this issue of the Journal, we read about two such initiatives.

The first venture involves the provision of a SAFE (Safer Anesthesia From Education) Obstetric course in Kigali, Rwanda. ${ }^{2}$ This course, which was originally created by Kate Grady from the UK, has been running in various venues for about three years. It has been very heavily supported by the Association of Anaesthetists of Great Britain and Ireland as well as by the WFSA. The aim of the course is to improve the quality and safety of obstetric anesthesia along with the management of critical complications that are often encountered by the attendees in their day-to-day work. There are lectures, discussion groups, and breakout sessions and a great deal of hands-on practical activity with a number of high-quality faculty and course coordinators. The course has in-built evaluation which takes place in the months following completion of 
the course. Local staff members who have trained on previous courses are encouraged to be heavily involved in the planning and organization of subsequent courses and eventually to take over the full management. This is the classical "teach the teachers" model that works very well for this course which encompasses all that is good about training in LMICs - it is a true quality tool.

Other high-quality practical learning adjuvants can be added into the basic course. For example, in Uganda, a Lifebox ${ }^{\circledR}$ teaching program that incorporates use of the WHO surgical checklist was recently added to the SAFE Obstetric course. ${ }^{3}$ Courses similar to SAFE Obstetric are now being developed for pediatric practice, and a third development is planned, namely, a shorter (probably oneday) general course on basic safe conduct within an operating room.

The second initiative is outlined in a letter in this issue of the Journal from a group from Halifax, Nova Scotia who were also supported by CAS IEF, in this case, to run a difficult airway workshop in Kigali. ${ }^{4}$ Again, this was a course which taught a basic set of skills and also enabled local staff to become teachers for future courses. Part of their course, however, was based on teaching a skill, blind nasal intubation, on mannequins. In my view, this technique is somewhat more controversial. The reference ${ }^{5}$ used to justify this approach provides little, if any, evidence to advocate this technique, and I did not find other strong references to recommend its reintroduction into modern airway management. I use the word "reintroduction" carefully. As I see it, this is arguably a somewhat antiquated airway technique and, as such, is no longer advocated by any national or international difficult airway algorithm. In my time as a trainee in the UK in the 1970s, this was an accepted technique that often utilized an added low percentage of carbon dioxide to facilitate the respiratory drive and cause the patient to "suck in the tracheal tube"! During that time period, there was often no monitoring of any type except for a blood pressure cuff.

The authors fully realize and refer to the limitations of a technique taught on mannequins. Even so, their accentuation of the advantages that this approach offers through its simplicity of setup and low cost has questionable relevance to issues of quality or the potential hazards of transferring classroom skills into the operating room.

In my view, there is insufficient evidence to substantiate blind nasal intubation as a suitable technique to teach in LMICs, and it certainly calls into question the quality attributes. Around the world, there appears to be an accepted rationale that it is "all right" to teach skills now regarded as "obsolete" in the affluent world to those who live in LMICs. The rationale is sometimes based on the premise that where people have very little, it is permissible and probably advantageous to teach old skills that were taught when the affluent world was less sophisticated. However, we are supposed to be living in an evidencebased world. A technique that once worked in the hands of people who, for example, performed the technique ten times a day, five days a week for over ten months a year in a different era does not necessarily transfer to the hands of an untrained novice today.

If my wife or daughter were pregnant and about to deliver in a hospital in a LMIC today, I would be reassured knowing that their caregivers had experienced a series of SAFE Obstetric courses. If they required a general anesthetic, I would not feel that same reassurance knowing that their caregivers had tried blind nasal intubation on a mannequin in a classroom. This is surely the test we should apply to all teaching programs around the world - i.e., would we be happy for our loved ones to undergo treatment by those who had studied that course? If our answer is "yes", then fine, let us promote and develop the teaching of that skill. On the other hand, if our answer is "no", then we should not be teaching or advocating the skill and we need to develop a better quality system.

\section{Fournir des soins de qualité en anesthésie dans les pays à revenus faibles ou intermédiaires}

En ce moment, un écart majeur existe dans la disponibilité des chirurgies essentielles et d'une anesthésie sécuritaire entre les populations des nations riches et de celles vivant dans des pays à revenus faibles ou intermédiaires (PRFI). On estime que seulement $3 \%$ des 234 millions d'interventions chirurgicales pratiquées chaque année dans le monde sont réalisés au sein du tiers le plus pauvre de la population mondiale. En fait, approximativement 2 milliards de personnes n'ont absolument aucun accès à la chirurgie.

De nombreuses organisations consacrent des sommes d'argent considérables, beaucoup de temps et d'efforts personnels pour tenter de trouver un moyen d'inverser ces statistiques stupéfiantes. Quelques-uns de ces groupes (et on compte maintenant une constellation de groupes de ce type, d'origine religieuse, universitaire ou non gouvernementale) assurent des interventions chirurgicales qui sont grandement nécessaires, souvent sur une base cyclique. Certaines de ces visites donnent presque un sentiment de « tourisme médical» sans bénéfice apparent à long terme pour la communauté en dehors du bénéfice très réel apporté à ceux dont les vies ont été transformées pour le mieux. 
En outre, procurer uniquement des incitatifs économiques pour créer de meilleurs soins ne fonctionne pas toujours. Un récent éditorial du New England Journal of Medicine a souligné une tentative des autorités indiennes pour tenter d'améliorer les soins et diminuer la mortalité maternelle en mettant en œuvre le programme Janani Suraksha Yojana où les mères recevaient de l'argent pour les inciter à accoucher dans les hôpitaux. Les résultats de cette entreprise ont montré que, bien qu'il y ait eu une augmentation spectaculaire du nombre d'accouchements dans les hôpitaux, la mortalité maternelle est restée inchangée. Les auteurs y plaidaient pour que la qualité soit l'aspect principal des interventions et finissaient par une grande affirmation: «....Faire plus n'est pas faire mieux. Faire mieux est mieux ». ${ }^{1}$

La Fédération internationale des sociétés d'anesthésiologistes (WFSA) soutient avec passion l'idée qu'au-delà d'apporter des succès individuels pour des cas chirurgicaux, tout enseignement et aide humanitaire doit inclure une composante majeure qui procure un legs et une durabilité au profit des populations locales. La Fondation internationale pour l'éducation de la Société canadienne des anesthésiologistes (CAS IEF) partage ce point de vue et a été très active en Afrique et au Népal au cours de ces dernières années. Nous pouvons lire le récit de deux de ces initiatives dans le présent numéro du Journal.

La première initiative concerne l'apport d'un cours d'obstétrique «SAFE» (Safer Anesthesia From Education) à Kigali (Rwanda). ${ }^{2} \mathrm{Ce}$ cours, initialement créé par Kate Grady (du Royaume-Uni), a été donné à différents endroits depuis environ trois ans. Il a été fortement soutenu par l'association des anesthésiologistes de Grande-Bretagne et d'Irlande (Association of Anaesthetists of Great Britain and Ireland) ainsi que par le WFSA. Le but de ce cours est d'améliorer la qualité et la sécurité de l'anesthésie obstétricale, ainsi que la gestion des complications critiques auxquelles sont souvent confrontés les intervenants dans leur travail de tous les jours. Il comporte des cours théoriques, des groupes de discussion et des sessions en petits groupes, ainsi qu'un grand nombre d'ateliers pratiques guidés par plusieurs coordonnateurs de cours et universitaires hautement qualifiés. Le cours est construit pour inclure une évaluation qui a lieu dans les mois suivant son achèvement. Les membres du personnel sur place qui ont déjà bénéficié d'une formation lors de cours précédents sont encouragés à s'impliquer largement dans la planification et l'organisation de cours ultérieurs et, ultimement, à en assurer la pleine gestion. Il s'agit d'un modèle classique «d'enseignement des enseignants » qui fonctionne très bien pour ce cours qui combine tout ce qui est bon concernant la formation dans les PRFI: il s'agit d'un véritable outil de qualité.
D'autres suppléments à un apprentissage pratique de haute qualité peuvent être ajoutés à ce cours de base. En Ouganda, par exemple, un programme d'enseignement Lifebox ${ }^{\circledR}$ qui incorpore l'utilisation de la liste de contrôle chirurgicale de l'OMS a été récemment ajouté au cours d'obstétrique «SAFE ». ${ }^{3}$ Des cours semblables aux cours d'obstétrique «SAFE » sont maintenant élaborés pour la pratique pédiatrique et un troisième développement est prévu: il s'agira d'un cours de portée générale, plus court (probablement sur une seule journée) sur le comportement sécuritaire de base au sein d'une salle d'opération.

La deuxième initiative est décrite dans une lettre publiée dans ce numéro du Journal et émanant d'un groupe de Halifax (Nouvelle-Écosse) qui était également soutenu par le CAS IEF: dans ce cas, il s'agissait de mener un atelier sur la prise en charge des intubations difficiles à Kigali. ${ }^{4}$ Ici encore, ce cours enseignait des ensembles d'habiletés de base et permettait également au personnel sur place de devenir les enseignants pour des cours ultérieurs. Néanmoins, une partie de leur cours reposait sur l'enseignement d'une technique, l'intubation nasale à l'aveugle, sur des mannequins. Selon moi, cette technique est plus controversée. La référence ${ }^{5}$ utilisée pour justifier cette démarche n'apporte que peu ou pas de données probantes pour défendre cette technique et je n'ai trouvé aucune référence solide pour recommander sa réintroduction dans la gestion moderne des voies respiratoires. Le recours au mot « réintroduction » est ici prudent. Tel que je le conçois, il s'agit on ne peut dire d'une technique de gestion des voies respiratoires plutôt dépassée et, en tant que telle, qui n'est plus, préconisée dans aucun algorithme, national ou international, d'intubation difficile. Lorsque j'étais en formation au Royaume-Uni, dans les années 1970, cette technique généralement acceptée utilisait souvent un faible pourcentage ajouté de dioxyde de carbone pour faciliter la stimulation respiratoire et faire en sorte que le patient « aspirait la sonde trachéale »! À cette même époque, il n'y avait souvent aucun type de monitorage, en dehors du brassard de pression sanguine.

Les auteurs ont pleinement conscience des limites d'une technique enseignée sur des mannequins et $\mathrm{y}$ font référence. Mais, même dans ce cas, leur insistance sur les avantages que cette approche offre grâce à la simplicité de son organisation et à son faible coût soulève des questions quant à sa pertinence en termes de qualité ou de risques potentiels, lorsque l'on envisage le transfert des habiletés acquises de la classe à la salle d'opération.

Il n'y a pas, à mon avis de données probantes suffisantes pour soutenir que l'intubation par voie nasale, en aveugle, est une technique qu'il convient d'enseigner dans les PRFI et cela soulève certainement des interrogations sur les caractéristiques de qualité. Mondialement, il semble exister 
une logique acceptée selon laquelle il n'y a pas de problème à enseigner dans les PRFI des habiletés maintenant considérées comme obsolètes dans le monde développé. Cette logique repose parfois sur la prémisse que là où les gens n'ont que très peu de moyens, il est permis et sans doute bénéfique d'enseigner de vieilles techniques qui étaient enseignées lorsque le monde développé était moins sophistiqué. Nous sommes cependant censés vivre dans un monde reposant sur des données probantes. Une technique qui fonctionnait entre les mains de professionnels qui, par exemple, la pratiquaient dix fois par jour, cinq jours par semaine et pour plus de dix mois par an à une autre époque n'est pas nécessairement transférable entre les mains de novices sans formation aujourd'hui.

Si j'avais une épouse ou fille enceinte et sur le point d'accoucher aujourd'hui dans un hôpital d'un PRFI, cela me rassurerait de savoir que les membres du personnel de soins ont suivi une série de cours d'obstétrique «SAFE ». Si elles avaient besoin d'une anesthésie générale, je ne me sentirais pas aussi rassuré sachant que les membres du personnel de soins ont essayé une intubation nasale à l'aveugle sur un mannequin en classe. C'est certainement le test que nous devrions appliquer à tous nos programmes d'enseignement dans le monde entier, à savoir serions-nous heureux si les personnes que nous aimons reçoivent un traitement de la part de ceux qui ont étudié ces cours? Si notre réponse est « oui », alors c'est parfait, encourageons et développons l'enseignement de ces connaissances. En revanche, si notre réponse est « non », nous ne devrions pas alors enseigner ou promouvoir ces connaissances et nous devons développer un meilleur système de qualité.

Conflict of interest None declared.

Conflit d'intérêts Aucun déclaré.

\section{References}

1. Scott $K W$, Jha $A K$. Putting quality on the global health agenda. N Engl J Med 2014; 371: 3-5.

2. Livingston P, Evans $F$, Nsereko E, et al. Safer obstetric anesthesia through education and mentorship: a model for knowledge translation in Rwanda. Can J Anesth 2014; 61: this issue. DOI: 10.1007/s12630-014-0224-8.

3. Finch LC, Kim RY, Ttendo S, et al. Evaluation of a large-scale donation of Lifebox pulse oximeters to non-physician anaesthetists in Uganda. Anaesthesia 2014; 69: 445-51.

4. Zhang J, Lamb A, Hung O, Hung C, Hung D. Blind nasal intubation: teaching a dying art. Can J Anesth 2014; 61: this issue. DOI: $10.1007 / \mathrm{s} 12630-014-0223-9$.

5. Law JA, Broemling N, Cooper RM, et al. The difficult airway with recommendations for management - part 2 - the anticipated difficult airway. Can J Anesth 2013; 60: 1119-38. 